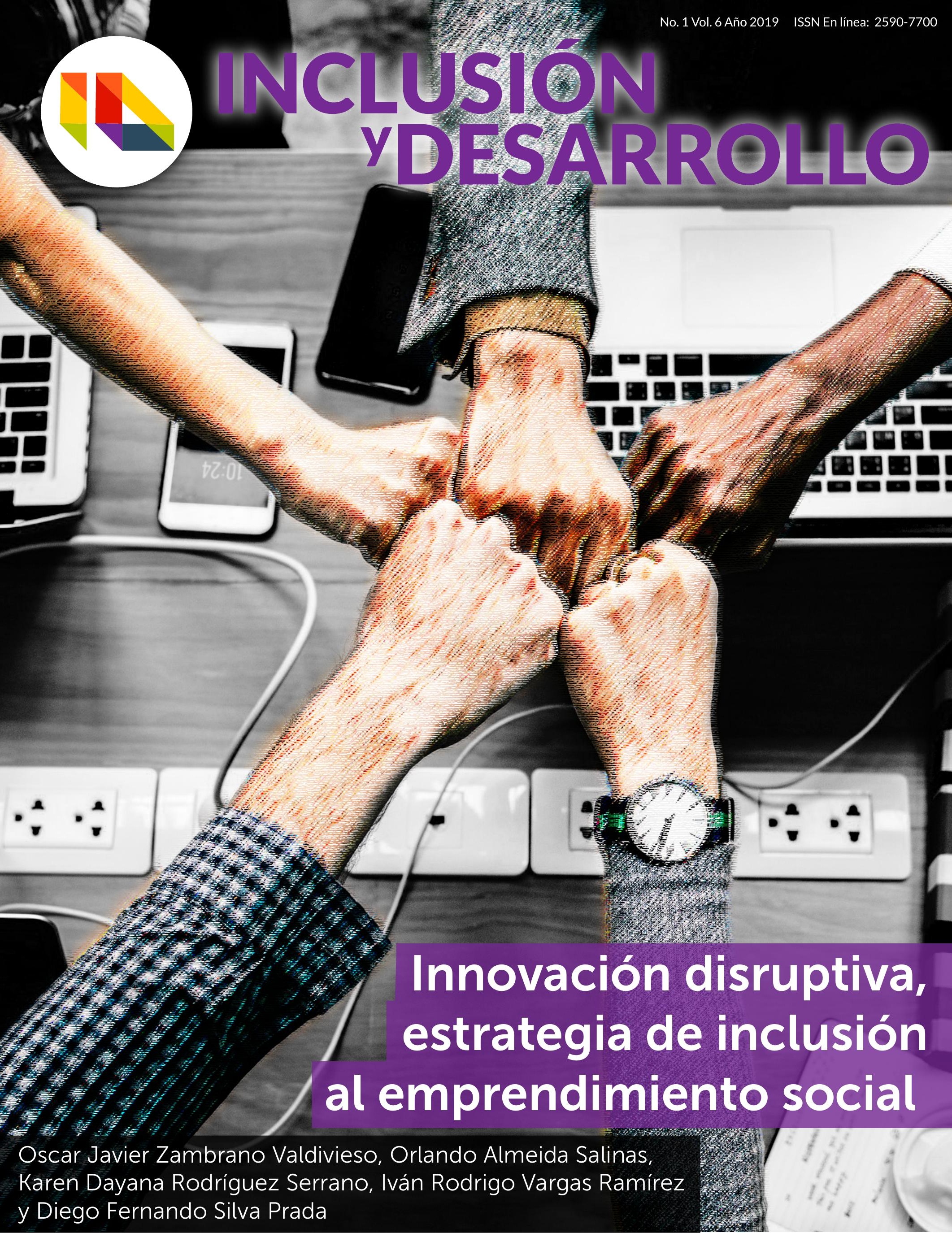




\section{ESTRATEGIA DE INCLUSIÓN AL EMPRENDIMIENTO SOCIAL}

\section{DisRUPTIVE INNOVATION, INCLUSION STRATEGY TOWARDS SOCIAL}

Oscar Javier Zambrano Valdivieso
ozambranov1@uniminuto.edu.co
Bucaramanga - Colombia
Orlando Almeida Salinas
Corporación Universitaria Minuto de Dios- UNIMINUTO
oalmeidasal@uniminuto.edu.co
Bucaramanga - Colombia
Corporación Universitaria Minuto de Dios- UNIMINUTO
Karen Dayana Rodríguez Serrano
karen.rodriguezs@uniminuto.edu
Universidad Manuela Beltrán
Bucaramanga - Colombia
Iván Rodrigo Vargas Ramírez
msc.ivanvargas@gmail.com
Bogotá D.C. - Colombia
Diego Fernando Silva Prada
dsilva@uniminuto.edu
Asociación para el avance de le ciencia ACAC
Bucaramanga - Colombia

\section{Resumen}

El presente artículo de revisión, surge de un marcado interés por analizar los procesos de innovación disruptiva como una estrategia de inclusión hacia el emprendimiento social; en cuanto al desarrollo metodológico, se realizó una revisión documental de forma sistemática en bases indexadas, con el fin de consultar e identificar información pertinente y de interés en la recopilación y construcción de los referentes teóricos necesarios para dar respuesta a la siguiente pregunta que guía la investigación: ¿Por qué analizar los procesos de innovación disruptiva como una estrategia de inclusión hacia el emprendimiento social? La investigación tiene un enfoque de tipo documental cualitativo, con alcance descriptivo - explicativo, y diseño basado en la teoría fundamentada.

Palabras clave: innovación, innovación disruptiva, estrategia, inclusión, emprendimiento social. 


\section{Abstract}

This review article, arises from a marked interest in analyzing the processes of disruptive innovation as an inclusion strategy towards social entrepreneurship. Regarding to the methodological development, a documentary review was carried out systematically, in order to consult and identify pertinent and interesting information in the compilation and construction of the theoretical references needed to answer the following question that guides this research: Why analyzing the processes of disruptive innovation works as an inclusion strategy towards social entrepreneurship? The research has a qualitative documentary approach with descriptive - explanatory scope, and design based on grounded theory.

Keywords: innovation, disruptive innovation, strategy, inclusion, social entrepreneurship. 


\section{INTRODUCCIÓN}

El presente artículo, busca analizar ¿̇por qué la innovación disruptiva se convierte en una estrategia de inclusión hacia el emprendimiento social?, siendo las empresas y las organizaciones el entorno en donde materializan conceptos como innovación, innovación social, innovación disruptiva, emprendimiento y emprendimiento social; los cuales son, en parte, posiblemente los garantes para minimizar las problemáticas emergentes en determinado sector del entorno productivo, dándoles la posibilidad de competir y evaluar el impacto económico y social que se genera en los diferentes mercados.

Es así cómo; mediante esta investigación, se demuestra que la innovación y sobre todo la innovación disruptiva, es una estrategia fundamental y necesaria en el mejoramiento continuo inclusivo de la sociedad.

Autores como Puelles \& Ezponda (2016) realizan una revisión del concepto de innovación, afirmando que éste ha sido estudiado desde la primera tercia en línea de tiempo del pasado siglo, partiendo desde las investigaciones realizadas por Schumpeter $(1934,1939$ y 1942) sobre la destrucción creativa; pero es de resaltar que en la primera parte del siglo XXI, las investigaciones alrededor de la innovación han logrado reconciliar no solo ciencias como la economía y la sociología de forma académica; sino también, y como lo afirman Candía, Coliñanco, Caro \& Hernández (2014), políticas económicas y sociales de estados y entidades supranacionales.

Vista desde la teoría, la innovación en el entorno empresarial se ha desarrollado con distintos enfoques, representantes y seguidores. Entre los trabajos pioneros se encuentran los de Marshall (1961 y 2009), quien llevó conceptos como la "teoría de la firma al centro del análisis económico y consideró determinante la distinción entre firma innovadora y firma optimizadora", siendo esta última la más relevante, por la pérdida de la capacidad innovadora de las empresas con el paso del tiempo.

Schumpeter (1954 y 1982), formalizó el término del "empresario innovador", quien logra posicionar en el mercado nuevos productos o implementar nuevos procesos en la producción mediante nuevas combinaciones de recursos productivos. Para este autor, la innovación es el fenómeno esencial del desarrollo económico; según él, "las firmas innovadoras pueden desafiar a las firmas optimizadoras y, por ende, impulsar el desarrollo económico". Uno de los enfoques de la teoría de la innovación y del desarrollo económico en que más se ha profundizado en los últimos años -directamente relacionado con el modelo interactivo- es el estudio de los sistemas nacionales y regionales de innovación. Al respecto, se destacan aportes teóricos de Penrose (1959), Chandler (1962) y Nelson \& Winter (1982), (Freeman, 1987; Dosi, 1989; Porter, 1990; Lundvall, 1992; Nelson, 1993; Edquist, 1997; Koschatzky, 1997), Abernathy y Utterback (1978), que podría definirse como «la red de instituciones, del sector privado y público, cuyas actividades e interacciones inician, importan, modifican o divulgan nuevas tecnologías» (Freeman, 1987), permitiendo que este sistema sea heterogéneo, abierto y dinámico, caracterizado por la escalabilidad y la retroalimentación efectiva.

Desde este sistema de redes, la innovación y el aprovechamiento de nuevas tecnologías, además de los aspectos particulares; dependen igualmente, de la sinergia e interacción entre ellos. La dinámica innovadora de una zona, no necesariamente está en relación de su infraestructura tecnológica (agregado de centros e instituciones que realizan actividades innovadoras) y de su objetivo cuantitativo en I+D (personal y gastos); sino también, de la actuación entre las empresas, administraciones públicas y otros agentes.

Macías (2011), en los últimos veinte años viene construyendo una perspectiva evolucionista, donde la innovación se posiciona como un sumario de aprendizaje que hace énfasis en el rol de lo humano para determinar cómo la empresa puede acumular capacidad innovativa y, por lo tanto, se añade explícitamente una dimensión social para trabajar en el aspecto de las capacidades. Precisamente la innovación es la capacidad que permite medir de la mejor forma la creación de valor de las empresas en temas de competitividad. (Tsai y Ghoshal, 1998).

La innovación permea y transforma los escenarios económicos o escuelas que desarrollan la teoría económica de la innovación, como lo demuestra Dahl (2001), (ver gráfico No 1). La competitividad empresarial, el crecimiento y el desarrollo económico, tal como lo plantea 
Sánchez \& Salazar (2010), lleva a preguntar por el ¿̇por qué estudiar la innovación?, ¿qué aporta la innovación a un estado, a un sector o a una empresa? Fagerberg (2005), arroja las siguientes ideas sobre estos interrogantes:

- La innovación implanta invenciones y, por tanto, diversidad en el medio económico; si no existiera innovación, la economía se situaría en un estado estacionario (Metcalfe, 1998) y no habría crecimiento.

- La innovación generalmente se concentra en algunos sectores productivos, los cuales presentan un crecimiento más ágil y repercuten en cambios estructurales en el mercado y, por ende, en las instituciones y es precisamente en la resiliencia de estas últimas frente a los cambios que se refleja en la sociedad el beneficio de la innovación.

- La innovación es un elemento con un fuerte dominio explicativo de los contrastes entre empresas, territorios o estados. Las empresas que innovan progresan, habitualmente más que aquellas que no lo hacen. Los territorios y estados que innovan ven cómo se fortalece su factor competitivo y su PIB (Producto Interno Bruto), por encima de quienes no lo hacen.

Medina \& Basurto (2018), demuestran como la innovación permite expandir y desarrollar nuevos mercados, generando cambios incrementales y disruptivos en soluciones que generalmente se establecen por avances en tecnología y mejores capacidades del mercado (Bower y Christensen, 1996). La innovación en la teoría consiste en una idea, práctica u objeto que es percibida por un individuo como algo nuevo. El concepto de innovación es puramente subjetivo dentro del individuo, y cuando la innovación se le comunica se encuentra con la opción de aceptarla o rechazarla. Una innovación implica en el individuo una nueva fuente de conocimiento (Anderson, Potočnik, \& Zhou, 2014; Cunningham \& Kwakkel, 2011; Pantano, 2014); como se aprecia en el Grafico No. 01 existen escuelas que forman la teoría económica de la innovación y permiten mediante su dinámica, la evolución a una innovación disruptiva en los procesos sistémicos.

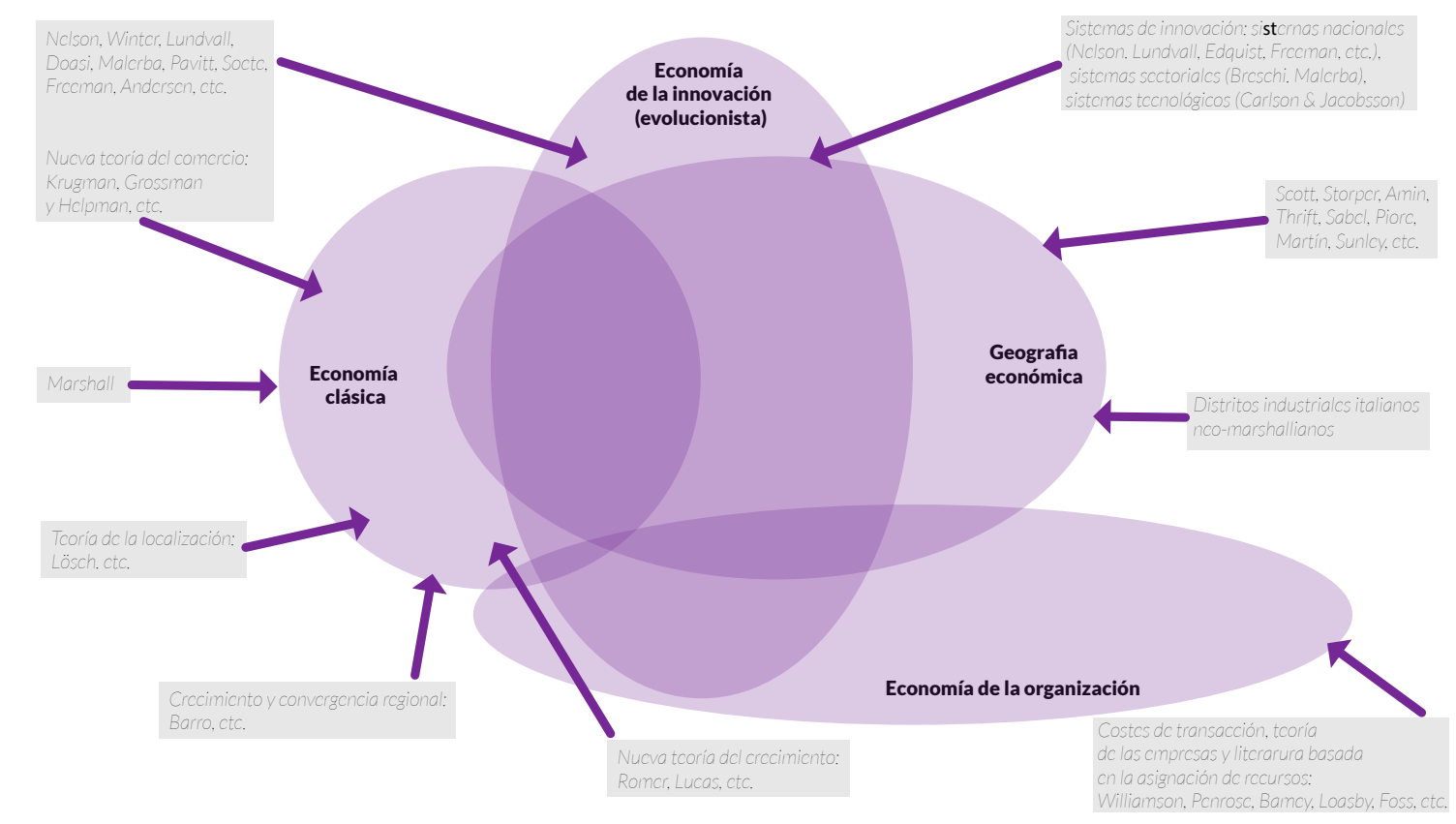

Grafico No. 1. Escuelas que forman la teoría económica de la innovación Tomado de: Dahl (2001). 


\section{Discusıón}

De acuerdo con Dávila (2015), el desempeño y la capacidad de gestionar la innovación incremental genera los puntos de diferenciación entre las organizaciones sociales; sin embargo, éstas deben planearse estratégicamente para impedir que no entreguen espacio para las innovaciones revolucionarias, por lo que ellas deben buscar maximizar la eficiencia, desarrollar actividades en torno a sus clientes y lograr altos niveles de creatividad que las proyectan a largo plazo.

Buckland \& Murillo (2014), señalan que, comparablemente al aumento del número de iniciativas para solucionar los inconvenientes sociales, germinan nuevos elementos de soporte para la innovación social y los emprendedores sociales. Entre los que se encuentran acciones en disímiles espacios, que parten desde la transformación del sector privado hasta las iniciativas gubernamentales que estriban a jóvenes emprendedores mediante programas educativos en planteles educativos de todo nivel e, inclusive, por medio de la incubación de proyectos nuevos. Es así, como, varias instituciones educativas ofertan actividades de investigación y programas de formación sobre innovación y sobre todo en el campo social. A lo anterior se debe incluir que los emprendedores sociales per se, apuestan por la proyección de iniciativas que apoyan al sector productivo con actividades de incubación y formación orientadas a generar espacios de coworking.

Murray, Caulier-Grice \& Mulgan (2010), consideran que la innovación social se estructura a partir de seis (6) etapas adaptadas por Carballo (2004), en el Gráfico No. 2. (Espiral de la innovación social), que inicia con la necesidad social y el diagnóstico del problema, analizando los grupos de innovación, la generación de pre proyectos, proyectos y propuestas, el desarrollo de un plan de acción que conlleve al diseño de prototipados y experimentación social y su respectivo modelo de negocio, y una estrategia de difusión social, que eventualmente puede llegar a generar un cambio sistémico, teniendo en cuenta que a cada etapa se le debe establecer su respectivo indicador para medir su dinámica e impacto.

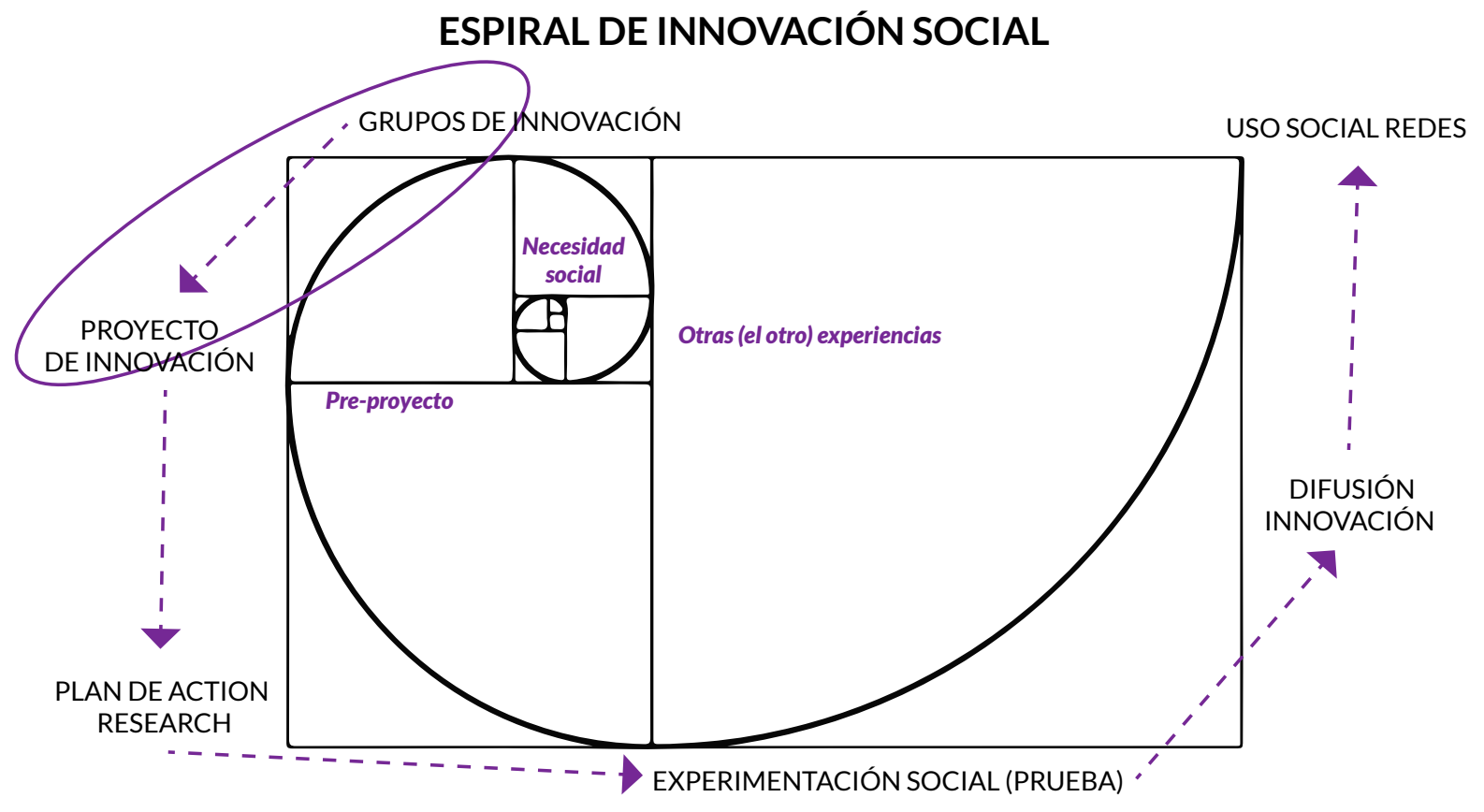

Grafico No. 2. Espiral de la innovación social Tomado de: Carballo (2004). 
Buckland \& Murillo (2013), proponen también un esquema acorde al Espiral de la Innovación Social de Carballo (2004), en donde priorizan las etapas de la Innovación Social y determinan cuáles serían los mecanismos para medir su impacto, entre las etapas que proponen está el diagnóstico y análisis; las propuestas e ideas; los prototipos; los prototipos y pruebas pilotos; el desarrollo del modelo de negocio; la ampliación y difusión y el cambio sistémico, como se aprecia en la Tabla 1. (Etapas de la Innovación Social y los mecanismos para medir su impacto).

Tabla 1. Las etapas de la Innovación Social y los mecanismos para medir su impacto.

\begin{tabular}{|c|c|}
\hline Etapa & Mecanismos de medición \\
\hline Diagnóstico y análisis & $\begin{array}{l}\text { Evaluación participativa; investigación aplicada; modelos } \\
\text { de pensamiento sistémico; peticiones en línea }\end{array}$ \\
\hline Propuestas e ideas & $\begin{array}{l}\text { Encuestas a los usuarios; votaciones online y wikis; bancos de ideas y buzones } \\
\text { de sugerencias; foros, asambleas y comisiones de ciudadanos }\end{array}$ \\
\hline Prototipos y pruebas piloto & $\begin{array}{l}\text { Pruebas controladas aleatorias; pruebas beta y pruebas abiertas; } \\
\text { prototipos lentos y rapidos; encuestas a usuarios }\end{array}$ \\
\hline $\begin{array}{l}\text { Desarrollo del modelo } \\
\text { de negocio }\end{array}$ & $\begin{array}{c}\text { Análisis de los ingresos y los resultados; evaluación de la inversión versus } \\
\text { el impacto social; cuadro de mando integral e informes de RSE }\end{array}$ \\
\hline Ampliación y difusión & $\begin{array}{l}\text { Matrices de contabilidad social; uso de franquicias y licencias; beneficio de } \\
\text { la inversión social; contabilidad de valor compartido; auditoría social }\end{array}$ \\
\hline Cambio sistémico & $\begin{array}{l}\text { Comparativa de resultados; existencia de nuevos sistemas de } \\
\text { producción, distribución, regulación y consumo }\end{array}$ \\
\hline
\end{tabular}

Tomado de: Buckland \& Murillo (2013).

Para Veiga (2016), la innovación disruptiva es un proceso por el cual una organización pequeña, con recursos limitados, logra tener la capacidad para desafiar y competir exitosamente con las organizaciones que ya son estables en el mercado; convirtiéndose así, en objeto de análisis en las actuales décadas por autores del nivel de Charitou y Markides (2002), Gilbert (2003) y Chandy y Tellis (2000). En la Tabla 2. (Origen de la innovación disruptiva), se presenta el origen de este concepto:

Tabla 2. Origen de la innovación disruptiva.

\begin{tabular}{|c|c|c|}
\hline No & Autor & Publicación \\
\hline 1 & Clayton y Bower (1995) & "Tecnologías disruptivas: alcanzando la ola" \\
\hline 2 & Christensen (1997) & $\begin{array}{c}\text { "El Dilema del innovador. Cuando las nuevas tecnologías } \\
\text { causan el fracaso de las grandes firmas" }\end{array}$ \\
\hline 3 & Molina y Morales (2002). & "Industrial districts and innovation: the case of the Spanish ceramic tiles industry" \\
\hline 4 & $\begin{array}{l}\text { Belussi, Sammarra } \\
\text { and Sedita, (2008) }\end{array}$ & $\begin{array}{l}\text { "Industrial districts evolutionary trajectories: localized } \\
\text { learning diversity and external growth" }\end{array}$ \\
\hline 5 & Christensen (2013) & "La solución del innovador. Creando y sosteniendo crecimiento exitoso" \\
\hline
\end{tabular}

Fuente: elaboración propia de los autores con base en (Veiga, 2016) y (Molina, Martínez, Valiente, 2017). 
Para comprender este argumento es útil revisar el modelo de innovación disruptiva planteado inicialmente por Bower \& Christensen (1995), en el cual se dividen las innovaciones en dos categorías: sustentadoras y disruptivas. La colectividad de las innovaciones de servicios y productos son sustentadoras; proveen una conspicua funcionalidad y calidad anexa a los usuarios más estrictos de una institución. Resaltando que algunas innovaciones sustentadoras son servicios o productos más revolucionarios y otras son mejoras incrementales.

Para Cabrol \& Severin (2010), una innovación disruptiva es una solución nueva que ofrece respuesta a quienes, antes de su aparición, no tenían opciones disponibles. Para que opere efectivamente, su aparición debe cumplir con tres condiciones: precio accesible, calidad inicial suficiente y potencial de mejora continua.

Así mismo, Christensen, Baumann, \& Ruggles (2006), afirman que la innovación se puede dividir en dos dimensiones, las disruptivas y las sustentadoras, siendo las primeras aquellas que suministran una mejor funcionalidad o calidad plus a los clientes más exigentes de una organización, y las segundas examinan como compensar al cliente reduciendo algunas capacidades o funciones de los servicios y productos de la innovación sustentadora. A nivel social se encuentran cinco cualidades de la innovación disruptiva a partir de lo estructurado por Christensen, Baumann, \& Ruggles (2006), como se evidencia en el Gráfico No. 3. (Innovación Disruptiva en el Sector social).

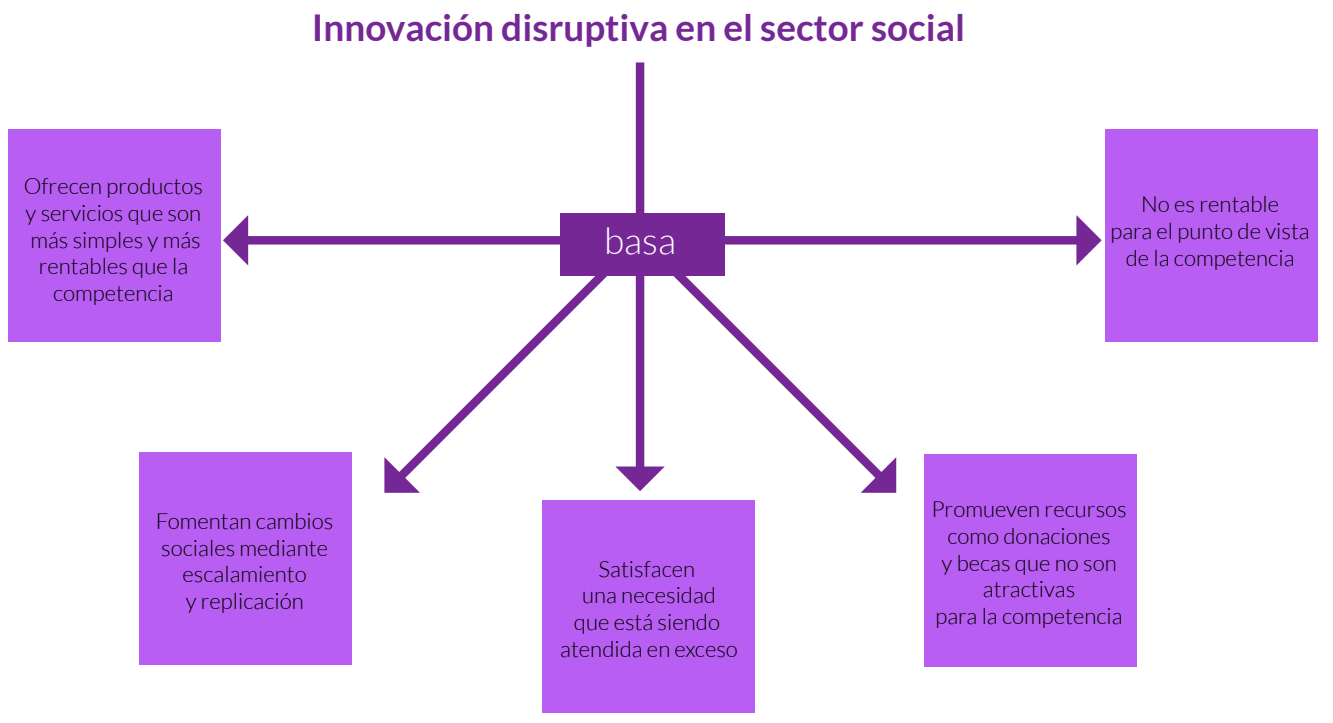

Grafico No. 3. Innovación Disruptiva en el Sector social.

Fuente: elaboración propia de los autores (2018), con base en (Christensen, Baumann, \& Ruggles, 2006).

En la obra Innovación disruptiva para el cambio social de Christensen, Baumann, Ruggles \& Sadtler (2006), se comprende la aplicación del modelo de innovación disruptiva a servicios y productos comerciales, conocida como innovación catalítica, en donde el cambio social es la meta principal y se debe considerar que los innovadores catalíticos comparten cinco cualidades:

“1. Generan cambios sociales sistémicos mediante el escalamiento y la replicación.
2. Satisfacen una necesidad que está siendo atendida en exceso (porque la solución existente es más compleja de lo que mucha gente requiere) o no atendida en absoluto. 3 . Ofrecen productos y servicios que son más simples y menos costosos que las alternativas existentes, y que podrían ser percibidos como de menor desempeño, pero que los usuarios consideran suficientemente buenos. 4. Generan recursos, tales como donaciones, becas, voluntarios o capital intelectual, de maneras que inicialmente son poco atractivas para los 
competidores establecidos. 5. Son a menudo ignorados, menospreciados o incluso alentados por actores establecidos para quienes el modelo de negocios no es rentable ni atractivo y, por tanto, evitan o se retiran del segmento de mercado". (Christensen, Baumann, Ruggles \& Sadtler, 2006, p.8).

Ahora, el concepto de innovación frugal se define como la intercepción de tres dinámicas de cambio, como lo expone Bhatti (2012) y se observa en el Gráfico N. 4. (Innovación frugal, sustentabilidad e inclusión social); el cambio tecnológico i), que sigue los patrones schumpeterianos de "destrucción creativa", es decir, la innovación tecnológica que investiga ventajas competitivas a través del desarrollo de nuevos procesos y productos (Schumpeter, 1934); la innovación social, ii), que busca incluir los consumidores/ciudadanos marginalizados por las modernas sociedades industrializadas (George, Macgahan y Prabhu, 2012); y iii) la innovación institucional que busca adaptar de manera eficiente las instituciones a un entorno global siempre cambiante. Esta intercepción tiene muchas implicaciones para el futuro de la sustentabilidad global:

- La innovación frugal de hecho favorece la optimización de recursos, sea a nivel local desde el micro y pequeñas empresas como en las grandes empresas transnacionales.

- Favorece el empoderamiento y la democratización de tecnología por parte de los usuarios introduciendo el concepto de "límite" en el empleo de recursos naturales.

- Revitaliza y redescubre el valor de la sabiduría tradicional que en la suma de los intervalos está fundada en prácticas que preservan el medioambiente.

- Favorece una disminución del impacto sobre el medioambiente: menos energía, menos desechos, menos consumo, menos recursos;

- Favorece la inclusión de los componentes sociales más desfavorecidos por el proceso productivo industrial, dándoles voz y posibilidades de desarrollo.

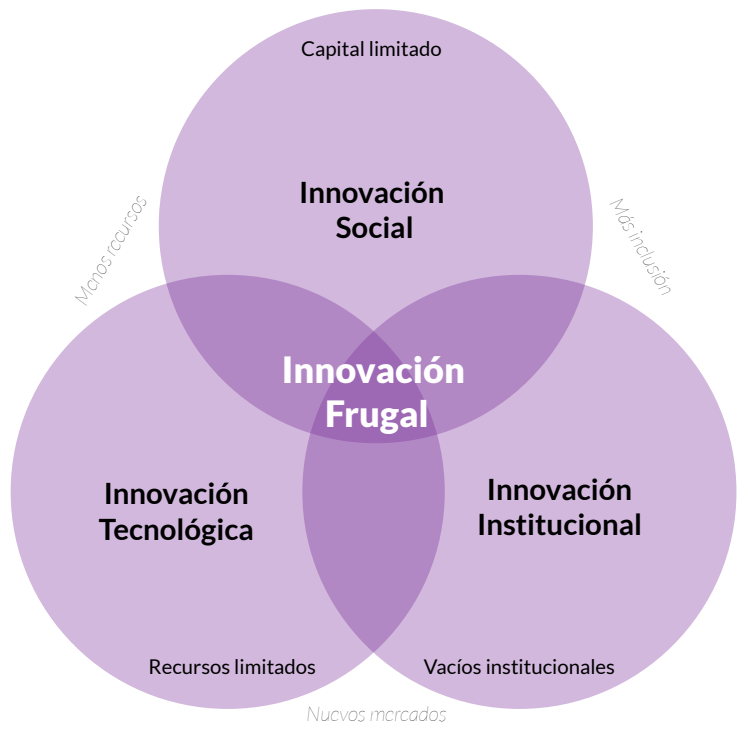

Grafico No. 4. Innovación frugal, sustentabilidad e inclusión social. Fuente: tomado de Bhatti (2012).

Para acuñar la definición de sustentabilidad global se debe aclarar que el término sustentable es sumado al término de desarrollo resultando implícito su prolongación en el tiempo. Pensadores como Barbier (1987) y Lélé (1991), formalizan el concepto de desarrollo como algo considerablemente indeterminado pero que ha ido progresando en el tiempo. Pero, como Harboth (1991) indica, la noción de desarrollo sustentable proyecta alcanzar dos objetivos:

"El concepto de desarrollo sustentable como objetivo presume dos cosas: primero, implica que también hay objetivos y caminos de de- 
sarrollo - por supuesto, se suponen aquellos puestos en práctica- que no pueden perdurar por largo plazo por amenazar tarde o temprano las bases ecológicas de la tierra. Segundo el 'desarrollo' bajo ningún criterio se abandona como objetivo, y para que el concepto no sea contradictorio con sí mismo, un desarrollo diferente al que hemos tenido hasta ahora, un desarrollo sustentable ecológica y socialmente, es concebible y practicable" (Harboth, 1991, p.9).

Se parte entonces de la discusión en torno al desarrollo sustentable que tuvo su foco en la sustentabilidad ecológica la cual hace referencia a la pérdida de valores ecológicos (respeto por el entorno, la biodiversidad, la herencia cultural, el equilibrio ambiental, etc.), una muestra de esta perspectiva puede ser tomada de lo enunciado por Pearce y Tuner:

“QQué significaría para una economía ser manejada de forma 'sustentable'? Como en el ejemplo de la pesca y los bosques, es tanto el producto de la economía que debe sustentarse, como el recurso que está en la base y que permite crear el producto. Sustentabilidad significa asegurarse que productos sustitutos son asequibles en la medida en que los recursos no renovables se vuelven físicamente escasos, y significa asegurar que los impactos ambientales resultado del uso de dichos recursos se mantengan dentro de la capacidad de soporte de la Tierra para asimilarlos" (Pearce; Turner, 1993, p.3-4).

La segunda polémica es entorno a la sustentabilidad social, considerada por lo general como la pobreza y el incremento de la tasa de natalidad en los países subdesarrollados. Como explica Lélé "la sustentabilidad ecológica no sólo tiene causas técnicas, sino también sociales: Para dar un ejemplo prototípico, uno puede decir que la erosión del suelo que mina las bases agrícolas de la sociedad humana es un caso de (in) sustentabilidad ecológica. Puede ser causado por el cultivo en tierras marginales sin las medidas adecuadas de conservación - la causa ecológica. Pero el fenómeno de la marginalización de los campesinos puede tener raíces sociales, que serían, entonces, las causas sociales de la sustentabilidad ecológica" (Lélé, 1991, p.610). En este precepto es imperiosa la dependencia directa con la inclusión social, reconociendo que es un concepto multifacético y de discusión constante en la literatura pues, en general, no hay un pacto con relación a cuáles son los elementos constitutivos del concepto, cuáles son sus entornos de aplicación y cual su médula conceptual.

Es así como existen una multiplicidad de ponencias con relación a los medios para alcanzar la inclusión social; Álvarez (2016), expone un panorama doctrinal muy interesante al afirmar que hay quienes razonan de suma importancia el estatus de ciudadanía como factor de inclusión; para otros la inclusión transita por el empleo remunerado (Unión Europea, 2004; Paz-Fuchs, 2008 y Williams, 2009); otros pensadores rotulan la importancia de los derechos en el sumario de la inclusión (Atkinson y Hills, 1998; Buckmaster y Thomas, 2009; Subirats, 2010), y otros proveen un profuso peso al reconocimiento y respeto de las identidades y las diferencias (Young, 1989, Lister, 2000: 43-47; Sennett, 2000; Paz-Fuchs, 2008). Pero, en este compendio de conceptualización, queda aún un factor divergente al precisar el termino de inclusión social cuando se señala que el ámbito de inclusión es la sociedad, a manera de que las personas o grupos focos de inclusión, quedaran en un infinito social separado que desabastece a los individuos de intercambios e interacciones (BID, 2007: 7; Mascareño y Carvajal, 2015: 132).

En sucintas lo que se busca con la inclusión social para el presente documento es su aplicabilidad a la innovación disruptiva como estrategia de inclusión hacia el emprendimiento social, es concebir el concepto según lo planteado por Anda, de Aguinaga \& González (2010), en donde la inclusión social admite no sólo la declaración de los derechos de todos los individuos, sus parquedades sociales y el paso a los satisfactores para revestir éstas; también reclama presagiar otro tipo de necesidades, como sociales, culturales, personales ambientales, como son la revalidación del ser (identidad) y la pertenencia a su colectivo social.

Por otro parte, autores como Roberts \& Woods (2005), precisan el emprendimiento social como un proceso multidimensional cuyo aserto está basado en los efectos alcanzados. Los estudiosos conciben estar de acuerdo en que estos efectos inquiridos son "tratar necesidades sociales", pero alcanzar un asentimiento respecto al proceso para alcanzarlo parece ser más ambiguo. Con Van 
der Veen \& Wakkee (2004), el emprendimiento social logra ser determinado como la destreza de registrar circunstancias hacia la creación de valor social. Así, para concebir el emprendimiento se logra concebir como una estructura, donde este es un proceso, más que un acaecimiento, y la exploración de una oportunidad es céntrica, donde la oportunidad es un momento por hacer anhelado que demanda el preámbulo de nuevas materias primas e insumos, de servicios y bienes y de métodos de organización interinstitucionales.

A partir de Palacios (2010), el emprendimiento social y su primordial representación de estructura productiva, la empresa social, germina en el siglo XXI como estándar mixto y novedoso que se encauza en disipar problemas sociales de modo sustentable.

La organización del sistema capitalista y la competición por los recursos que se da intrínsecamente en éste, no consiente que las organizaciones sean cien por ciento no rentables sin que afronten inconvenientes en el corto plazo y terminen por desaparecer del mercado. Para Apetrei, Ribeiro, Roig \& Mas Tur (2013), el emprendedor social posee el objetivo elemental de crear valor social en lugar de riqueza personal; esta percepción es validada igualmente por Urbano, Toledano \& Soriano (2010) y por Zadek \& Thake (1997), en sus estudios que conllevan a entender que el emprendimiento social se genera mediante los procesos de innovación social y no mediante la innovación económica; tesis que es también acogida por Kramer (2005); Austin, Stevenson \& Wei-Skillern (2006); Leadbeater \& Wilsdon (2007) y que logran establecer un escenario en donde abordan problemas sociales en lugar de necesidades individuales, confirmando así lo establecido por Harding (2006) y en las investigaciones de Westall \& Chalkley (2007).

Finalmente, y a partir de sus objetivos, se logra definir al emprendedor social como un individuo que intenta solucionar un problema social con una perspectiva innovadora a la vez que desarrolla una dinámica emprendedora. A discrepancia del emprendedor encauzado a los negocios, el emprendedor social está, en primer lugar, encaminado a instaurar un impacto social auténtico y, en segundo lugar, a lograr la superación financiera.

\section{Materiales y MÉtodos}

Se realizó una revisión documental de forma sistemática, con el colofón de consultar e identificar información pertinente y de interés en la recopilación y construcción de los referentes teóricos necesarios para dar respuesta a la siguiente pregunta que guía la investigación ¿̇Porque analizar los procesos de innovación disruptiva como una estrategia de inclusión hacia el emprendimiento social, Colombia 2018?

De igual forma se formuló el siguiente objetivo general: Analizar los procesos de innovación disruptiva como una estrategia de inclusión hacia el emprendimiento social, Colombia 2018, a partir de la revisión documental.

\section{Contexto de la inVestigación}

\section{TIPO DE INVESTIGACIÓN}

La investigación se realizó bajo un enfoque de tipo documental cualitativo, teniendo en cuenta que presenta las siguientes características: con el fin de argumentar y soportar el planteamiento del problema y el reporte de resultados se realiza una revisión teórica; los resultados del estudio se presentan a manera de hipótesis de trabajo, sin que implique probar hipótesis de forma estadística; la recolección de datos consiste en obtener descripciones detalladas acerca de los procesos de innovación disruptiva como una estrategia de inclusión hacia el emprendimiento social, los investigadores llevan a cabo una revisión sistemática documental, de modo tal que permita la contextualización y conceptualización de los principales aspectos relacionados con el objeto de estudio. Lo anterior se encuentra acorde con lo propuesto por Hernández, Fernández \& Baptista (2014) para el desarrollo de este tipo de investigaciones.

El alcance de la investigación es descriptivo, ya que se encuentra orientada a describir, especificar, detallar las características y propiedades relacionadas con los procesos de innovación disruptiva como una estrategia de inclusión hacia el emprendimiento social, (Hernández, Fernández \& Baptista 2014). Así mismo, la revisión documental descriptiva según lo planteado por Merino-Trujillo (2011; Pág. 36) "proporciona al lector una puesta al día sobre conceptos útiles 
en áreas en constante evolución", siendo de gran utilidad en diversos campos de la enseñanza.

La población documental se encuentra conformada por referencias de uso y conocimiento científico universal, básicamente de artículos científicos especializados nacionales e internacionales, a partir de las cuales se indaga, interpreta y presentan datos sobre un tema determinado.

A su vez, Merino-Trujillo (2011), afirman que este tipo de artículos de revisión son realizan "estudios detallados, selectivos y críticos que examina la bibliografía publicada y la sitúa en cierta perspectiva". Se resalta que "no son una publicación original y su finalidad es realizar una investigación sobre un tema determinado, que concierne al problema de investigación abordado".

El diseño de esta investigación se encuentra basado en la teoría fundamentada, ya que, según Hernández, Fernández \& Baptista, (2014) utiliza procedimientos cualitativos para explicar de manera conceptual una acción. Así mismo, este tipo de diseño permite explicar temas que son de difícil comprensión o reestructurar fundamentos considerados como desactualizados o poco utilizados.

\section{Conclusiones}

La innovación es hoy por hoy considerada como una necesidad absoluta e imperiosa en todo entorno productivo; resaltando, la imperiosa necesidad que tienen las organizaciones al buscar soluciones innovadoras a las dificultades que surgen en la sociedad o en sus clientes perennemente o de lo contrario posiblemente el core del negocio no prosperará, su nivel de competitividad será mínimo e inciertamente serán relegadas en el mercado.

La innovación es comprendida como la diligencia de nuevas ideas, productos, servicios, prácticas y conceptos, con la finalidad de ser útiles para el aumento de la competitividad y la productividad. Un componente primario de la innovación es su diligencia triunfante de carácter lucrativo. No solo hay que ingeniar algo, sino igualmente, asentar en el mercado; recalcando que concurre un componente agregado, particular de la concepción existente de la innovación: un sumario de evolu- ción. Hoy en día, el reto radica en instaurar knowhows constituidos y metódicos en las estructuras productivas para alcanzar que perennemente provoquen efectos innovadores y triunfantes en la rígida prueba del mercado, y trasfieran a creación de una cultura de la innovación en el entorno.

Es de resaltar que las investigaciones alrededor de la innovación han logrado reconciliar no solo ciencias como la economía y la sociología de forma académica; sino también, desde la óptica de la formulación de políticas económicas y sociales de estados y entidades supranacionales.

Desde 1954, existe gracias a Schumpeter el término del "empresario innovador", definiéndolo como quien logra posicionar en el mercado nuevos productos o implementar nuevos procesos en la producción mediante nuevas combinaciones de recursos productivos.

Existe la disyuntiva de la innovación incremental que se define como los cambios dirigidos a incrementar la funcionalidad y las prestaciones de la empresa, pero que si se suceden de forma acumulativa pueden constituir una base importante de progreso y la innovación radical entendida como un rompimiento con lo ya determinado, nuevos procesos o productos que no logran entenderse como un progreso natural de los ya existentes. Se logran casi siempre como resultado de procesos definidos de I\&D en laboratorios (dentro o fuera de la empresa) e institutos especializados, son explosivas y fragmentan el statu quo.

Esta innovación radical conlleva al enfoque de la innovación disruptiva que se orienta en desarrollar soluciones simples a usuarios desatendidos por las empresas y organizaciones sociales; generando alternativas adecuadas a problemas sociales presentados tanto en escalas pequeñas como barrios, veredas hasta municipios, departamentos y escala nacional.

Para desarrollar emprendimiento social basado en innovación disruptiva es necesario que las organizaciones del Estado analicen y actualicen sus mecanismos de desarrollo para cubrir necesidades de la sociedad con base en la disminución de funciones, capacidades y estrategias que las empresas y organizaciones convencionales desarrollan, solo hasta este punto se lograra crear valores agregados que apunten a un mejor bienestar social 


\section{Referencias bibliográficas}

Abernathy, W. J., and Utterback, J. M. (1978): «Patterns of Industrial Innovation», Technology Review, 80(7), 40-47.

Álvarez, B. S. (January de 2016). ¿̇Inclusión en qué? Conceptualizando la inclusión social. Ehquidad International Welfare Policies and Social Work Journal, $\mathrm{N}^{\circ}$ 5. Obtenido de file://C:/Users/Karen\%2oRodriguez/Downloads/1333-3468-1PB.pdf

Anderson, N., Potočnik, K., \& Zhou, J. (2014). Innovation and creativity in organizations a state-of-the-science review, prospective commentary, and guiding framework. Journal of Management, 40(5), 1297-1333.

Apetrei, A., Ribeiro, D., Roig, S., \& Mas Tur, A. (2013). El emprendedor social-una explicación intercultural. CIRIECEspaña, Revista de Economía Pública, Social y Cooperativa, (78).

Austin, J., Stevenson, H. \& Wei-Skillern, J. (2003): "Social Entrepreneurship and Commercial Entrepreneurship: Same, Different, or Both?”, Working Paper Series, No. 04-029, Harvard Business School.

Barbier, Edward. The concept of sustainable economic development. Environmental Conservation, Lausanne: Elsevier Sequoia, v.14, n.2, 1987

Bayus, B. L., Griffin, A., and Lehmann, D. (1998): «From the Special Issue Editors», Journal of Product Innovation Management, 15(2), 108-110.

Belussi, F., Sammarra, A., and Sedita, S. R. (2008): «Industrial districts evolutionary trajec- tories: localized learning diversity and external growth», Artículo presentado en la 25th Celebration Conference 2008 sobre Entrepreneurship and innovation - organizations, in- stitutions, systems and regions, Copenhage.

Bhatti, Y. (2012): "What is Frugal, what is Innovation? Towards a Theory of Frugal Innovation", Social Science Research Network working papers. Disponible en: http://ssrn.com/abstract=2005910.

Bower, J. L., \& Christensen, C. M. (1995). Disruptive technologies: catching the wave.

Bower, J. L., \& Christensen, C. M. (1996). Disruptive technologies: Catching the wave. The Journal of Product Innovation Management, 1(13), 75-76.

Buckland, H.; Murillo, D. (2013). Vías hacia el cambio sistémico. Ejemplos y variables para la Innovación Social, Instituto de Innovación Social de ESADE.

Buckland, H., \& Murillo, D. (2014). La innovación social en América Latina. Marco conceptual y agentes. Instituto de Innovación social. ESADE. Recuperado en http://idbdocs. iadb. org/wsdocs/getDocument. aspx.

Cabrol, M., \& Severin, E. (2010). TICs en educación: una innovación disruptiva.

Candía, J. G., Coliñanco, L. G., Caro, C. L., \& Hernández, N. R. (2014). Estrategia y cultura de innovación, gestión de los recursos y generación de ideas: prácticas para gestionar la innovación en empresas. Pensamiento \& Gestión, (36), 107133. doi:10.14482/pege.36.5567.

Carballo, R. (2004): “En la Espiral de la Innovación” Ed. Díaz de Santos.

Cunningham, S. W., \& Kwakkel, J. (2011). Innovation forecasting: A case study of the management of engineering and technology literature. Technological Forecasting and Social Change, 78(2), 346-357. doi:http://dx.doi.org/10.1016/j. techfore.2010.11.001

Chandler, A. D. (1962). Strategy and structure: Chapters in the history of the American enterprise. Massachusetts Institute of Technology Cambridge.

Christensen, C., Baumann, H., Ruggles, R., \& Sadtler, T. (2006). Innovación Disruptiva para el Cambio Social. Harvard Business Review América Latina, diciembre.

Christensen, C. (2013). The innovator's dilemma: when new technologies cause great firms to fail. Harvard Business Review Press.

Christensen, Baumann, \& Ruggles, 2006) Christensen, C. M., Baumann, H., \& Ruggles, R. (2006). Innovaci\{ó\}n disruptiva para el cambio social Innovaci\{ó\}n disruptiva para el cambio social. Business.

Christensen, C. M., Baumann, H., \& Ruggles, R. (2006). Innovaci\{ó\}n disruptiva para el cambio social Innovaci\{ó\}n disruptiva para el cambio social. Business. 
Christensen, C. M., Raynor, M. E., \& McDonald, R. (2015). What Is Disruptive Innovation? Harvard Busines Review, 1(1), 75-76. Retrieved from https://hbr.org/2015/12/what-is-disruptive-innovation?referral=00205\&amp; $\mathrm{cm}$ mmc=email-_-newsletter-_-bestofissue-_-bestofissue_date\&amp;utm_source=newsletter_bestofissue\&amp;utm_ medium=email\&amp;utm_campaign=bestofissue_date

Dávila, A., \& Epstein, M. (2015). “empresa start-up.” Revista de Negocios IEEM, 49-51.

Dahl, M. S. (2001): What is essence of geographic clustering. Paper presented in the DRUID Nelson \& Winter Conference, 12-15 de junio, en Aalborg Denmark

de Anda, A. B. B., de Aguinaga Vázquez, P., \& González, C. Á. (2010). El trabajo colaborativo y la inclusión social. Apertura, 2(1), 48-59.

Fagerberg, J. (2005) Innovation: A guide the Literature. En: J. Faberberg, D. Mowery y R.R. Nelson (Eds). The Oxford Handbook of Innovation. Oxford University Press. Oxford; 1- 26.

George, G., Macgahn, A. y Prabhu, J. (2012): "Innovation for inclusive growth: towards a theoretical framework and a research agenda." Journal of Management Studies, vol. 49, no 4, pp. 662-683.

Gilbert, C. (2003): «The disruption opportunity», MIT Sloan Management Review, 44(4), 27- 33. JOUR.

Harboth, Hans-Jurgen. The debate about sustainable development: starting point for an environment-oriented international development policy? Economics, Tubingen: Institute for Scientific Co-operation, v.44, p.7-31, 1991.

Harding, R. (2006): “Social Entrepreneurship Monitor”, London Global Entrepreneurship Monitor.

Hernández, Fernández \& Baptista. (2014). Metodología de la Investigación sexta edición. México, editorial McGraw-Hill.

Kramer, M.R. (2005): "Measuring innovation: evaluation in the field of social entrepreneurship", A Publication for Skoll Foundation by Foundation Strategy Group

Lélé, S. M. Sustainable development: a critical review. World Development, Oxford: Pergamon Press, v.19, n.6, p.607-621, jun. 1991.

Leadbeater, C., \& Wilsdon, J. (2007). El Atlas de las ideas: cómo la innovación puede ser beneficiosa para todos.

Macías Cardona, H. A. (2011). Innovación y responsabilidad social: una reflexión sobre los puntos de encuentro. Universidad \& Empresa, (21).

Marshall, A. (1961). Principles of economics: An introductory volume. London: Macmillan.

Marshall, A. (2009). Principles of economics: unabridged eighth edition. Cosimo, Inc.

Medina, R. A. N., \& Basurto, C. A. M. (2018). Discusión de los distintos tipos de innovación. Revista Publicando, 5(15), 5972.

Metcalfe, (1998) Evolutionary Economics and Creative Destruction. London. Routledge.

Merino-Trujillo, A. (2011). Como escribir documentos científicos (Parte 3). Artículo de revisión. Salud en Tabasco, 17(1-2), 36-40.

Molina y Morales, F. X. (2002): «Industrial districts and innovation: the case of the Spanish ceramic tiles industry», Entrepreneurship \& Regional Development, 14(4), 317-335.

Murray, R.; Caulier-Grice, J.; Mulgan, G. The Open Book of Innovation. The Young Foundation y Nesta, 2010.

Nelson, R. R., \& Winter, S. G. (1982). The Schumpeterian tradeoff revisited. The American Economic Review, 72(1), $114-132$.

Palacios Núñez, G. (2010). Emprendimiento social: integrando a los excluidos en el ámbito rural. Revista de Ciencias Sociales (Ve), 16(4).

Pantano, E. (2014). Innovation management in retailing: From consumer perspective to corporate strategy. Journal of Retailing and Consumer Services, 21(5), 825-826. doi:http://dx.doi.org/10.1016/j.jretconser.2014.02.017

Pearce, D.; Turner, R. K. Defining sustainable development. In: PEARCE, David. Blueprint 3: measuring sustainable development. London: Earthscan, 1993. p.3-14.

Penrose, E. T. (1959). The theory of the growth of the firm. New York: Sharpe. 
Puelles, A. A., \& Ezponda, J. E. (2016). ¿Qué es la innovación social? El cambio de paradigma y su relación con el Trabajo Social/What is social innovation? The paradigm shifts and its relationship with Social Work. Cuadernos de Trabajo Social, 29(2), 163 .

Roberts, Dave and Woods, Christine (2005). Changing the world on a shoestring: The concept of social entrepreneurship. University of Auckland. Business Review. AUTUMN 2005. Pp. 45-51

Sánchez, M. P., \& Salazar, J. C. (2010). El papel de la innovación en el nuevo modelo económico español. Editado por Accenture SL en el marco de la Cátedra UAM-Accenture de Economía y Gestión de la Innovación.

Schumpeter, J. A. (1934): The theory of economic development: an inquiry into profits, capital, credit, interest, and the business cycle, Cambridge, Transaction Publishers Cambridge.

Schumpeter, J.A. (2008[1939]). Business Cycles. Nueva York, Toronto, Londres: McGraw-Hill. (Edición electrónica de D. Lagrange). Quebec, Canadá, 2008, 80 (consultado el 15-8-2011).

Schumpeter, J. A. (1975[1942]). Capitalism, Socialism and Democracy. (2a edición). Nueva York: Harper \& Row.

Schumpeter, J. A. (1954). History of economic analysis. Psychology Press.

Schumpeter, J. A. (1982). The Theory of Economic Development: An Inquiry into Profits, Capital, Credit, Interest, and the Business Cycle (1912/1934). Transaction Publishers. -1982. -January, 1, 244.

Tsai, W., and Ghoshal, S. (1998): «Social capital and value creation: The role of intrafirm net- works», Academy of Management Journal, 41(4), 464-478.

Urbano, D., Toledano, N. \& Soriano, D.R. (2010): “Analyzing social entrepreneurship from an institutional perspective: evidence from Spain”, Journal of social entrepreneurship, 1(1), 54-69.

Valdivieso, Ó. J. Z., Salinas, O. A., Uribe, E. A. S., \& Pineda, J. E. R. (2017). La enseñanza de la lengua de señas colombiana como estrategia pedagógica para la inclusión educativa-Estudio de caso-(The teaching of the Colombian language of signals as pedagogical strategy for educational inclusion, case study). Inclusión \& Desarrollo, 5(1).

Van der Veen M, Wakkee I. 2004. Understanding the Entrepreneurial Process. In: Watkins D (ed.). Annual Review of Progress in Entrepreneurship (ARPENT); pp. 114-52.

Westall, A., \& Chalkley, D. (2007). Social Enterprise Futures, London: The Smith Institute.

Zadek, S. \& Thake, S. (1997): Practical people, noble causes. How to support community-based social entrepreneurs, New Economics Foundation, London. 
Revista incluida en los siguientes agregadores de contenidos
Revista incluida en los siguientes directorios

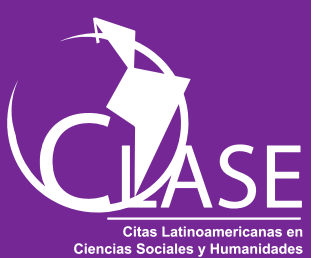

Ullbiblat

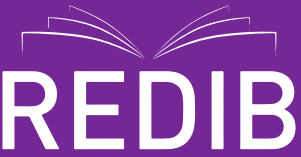

Red Iberoamericana

de Innovación y Conocimiento Cientifico

\section{Google Académico}
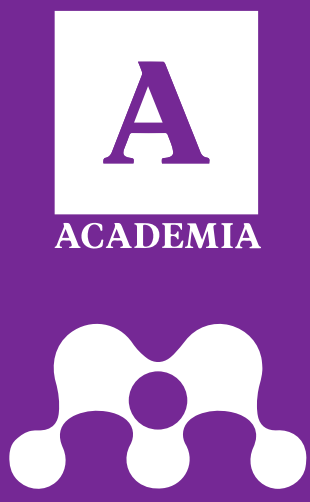

MENDELEY

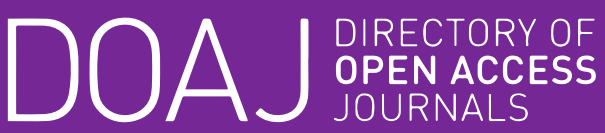

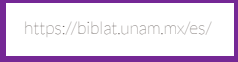
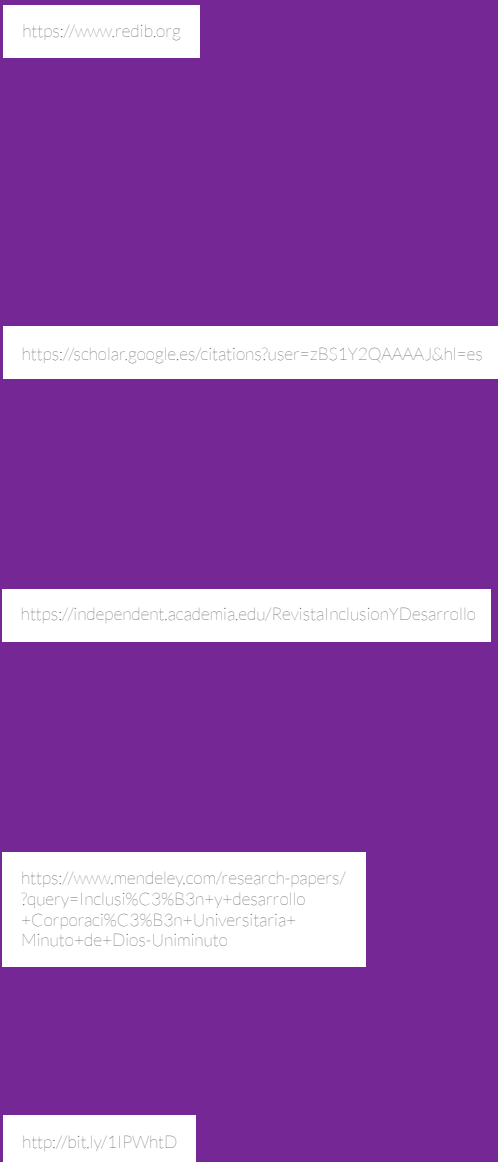

Revista incluida en la siguiente red social

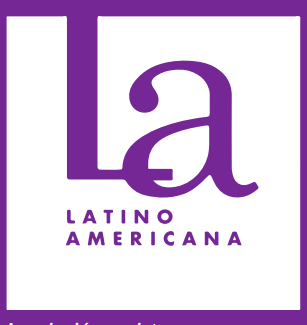



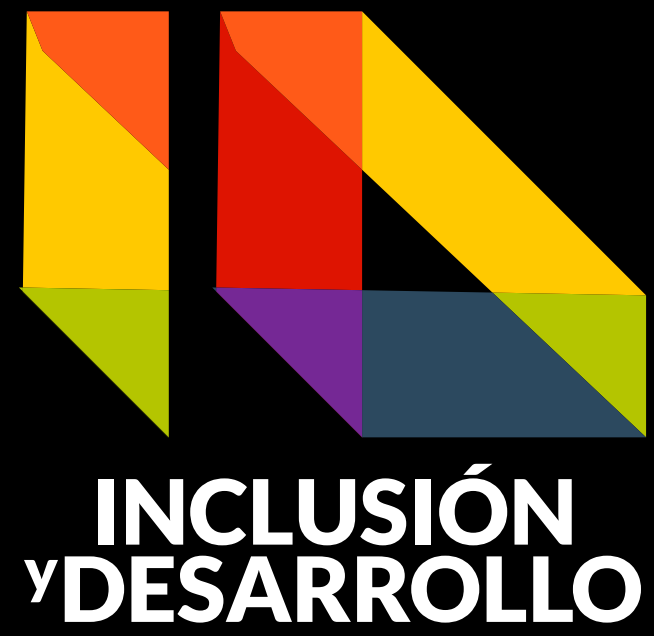

No. 1 Vol. 6 Año 2019 ISSN En línea: 2590-7700

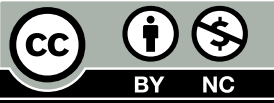

